\title{
Projecting syzygies of curves
}

\author{
Michael Kemeny
}

\begin{abstract}
We explore the concept of projections of syzygies and prove two new technical results. We firstly give a precise characterization of syzygy schemes in terms of their projections; secondly, we prove a converse to Aprodu's projection theorem. Applying these results, we prove a conjecture of G. Farkas and the author [Linear syzygies of curves with prescribed gonality, Adv. Math. 356 (2019), 106810] stating that extremal syzygies of general curves of non-maximal gonality embedded by a linear system of sufficiently high degree arise from scrolls. Lastly, we prove Green's conjecture for general covers of elliptic curves (of arbitrary degree) as well as a new result for curves of even genus and maximal gonality.
\end{abstract}

\section{Introduction}

Let $X$ be a projective variety and $L$ a line bundle, assumed to be very ample for simplicity. One of the most fundamental objects in algebraic geometry is the section ring

$$
\Gamma_{X}(L):=\bigoplus_{n \in \mathbb{Z}} H^{0}\left(X, L^{\otimes n}\right) .
$$

In order to understand the structure of $\Gamma_{X}(L)$, one treats it as a $\operatorname{Sym}\left(H^{0}(X, L)\right)$-module and takes the minimal free resolution. The syzygy spaces $K_{i, j}(X, L)$ are then the graded pieces which appear in the resolution. Their dimensions give important invariants $b_{i, j}(X, L)$ of the polarized variety $(X, L)$, known as Betti numbers.

Our understanding of the syzygies of $(X, L)$ is highly limited. At present, our knowledge is most complete in the case where $\operatorname{dim} X=1$, that is, $X$ is an algebraic curve $C$. Most of the known results are vanishing theorems, providing conditions for the vanishing of Betti numbers $b_{i, j}(C, L)$. Some highlights include Voisin's theorem on the generic Green's conjecture, which concerns the case $L=\omega_{C}$ (see [Voi02, Voi05]), as well as the recent proofs of the gonality conjecture [EL15] and the generic secant conjecture [FK16], which are concerned with the case of more general line bundles $L$. Whilst the gonality conjecture is known for arbitrary curves, the full statements for Green's conjecture and the secant conjecture are still very much open in the non-generic case.

More recently, work has been done on trying to go beyond vanishing theorems and describe certain values of $b_{i, j}(C, L)$ in terms of geometry. For example, the first linear Betti number

Received 3 November 2018, accepted in final form 18 October 2019.

2010 Mathematics Subject Classification 14H51, $13 \mathrm{D} 02$.

Keywords: curves, syzygies, Green's conjecture.

This journal is (C) Foundation Compositio Mathematica 2020. This article is distributed with Open Access under the terms of the Creative Commons Attribution Non-Commercial License, which permits non-commercial reuse, distribution, and reproduction in any medium, provided that the original work is properly cited. For commercial re-use, please contact the Foundation Compositio Mathematica.

The author is supported by NSF grant DMS-1701245. 


\section{KEMENY}

$b_{1,1}(C, L)$ describes the number of quadrics required to generate the ideal of $I_{C}$. It is not unreasonable to hope that other Betti numbers likewise carry geometric information. In [FK19] and [Kem18], attention was focused on the last of the non-zero linear Betti numbers $b_{g-k, 1}\left(C, \omega_{C}\right)$ for canonical curves of non-maximal gonality $k$. It was found that this extremal Betti number counts a classically studied invariant, namely the number of minimal pencils, that is, maps $f: C \rightarrow \mathbf{P}^{1}$ of degree equal to the gonality $k$ (up to genericity hypotheses)

The number of minimal pencils on a general $k$-gonal curve was first showed to be finite by Segre [Seg28]. More recently, Arbarello-Cornalba and Coppens have obtained several important results on the loci of curves carrying multiple minimal pencils; see [AC81, Cop88, Cop97]. In particular, a general curve of non-maximal gonality has a unique minimal pencil, and in this case, Schreyer has conjectured $b_{g-k, 1}\left(C, \omega_{C}\right)=g-k$, which both implies Green's conjecture $b_{g-k+1,1}\left(C, \omega_{C}\right)=0$ and gives a more geometric interpretation for it, as we explain below.

One of the goals of this paper is to prove a result interpolating and extending both the vanishing theorem of [EL15] and the verification of Schreyer's conjecture in [FK19].

Theorem 1.1. Let $C$ be a general $k$-gonal curve $C$ of genus $g \geqslant 2 k-1$ for $k \geqslant 4$. Let $L$ be an arbitrary line bundle on $C$ of degree $\operatorname{deg}(L) \geqslant 2 g+k$. Then

$$
b_{r(L)-k, 1}(C, L)=r(L)-k .
$$

In the result above, $r(L)=h^{0}(L)-1$. This result was conjectured in [FK19, Conjecture 0.5].

To explain the importance of Theorem 1.1 and illustrate the link to [EL15], we first reinterpret the statement geometrically. One of the only classes of varieties for which it is possible to determine the minimal free resolution are determinantal varieties described by the degeneracy locus of a morphism of vector bundles $\mathcal{V}_{1} \rightarrow \mathcal{V}_{2}$; see [Las78]. One strategy to study syzygies of a curve $C$ is to embed the curve into a determinantal variety $Z$ and then restrict the determinantal syzygies to $C$.

This strategy was employed in Voisin's proof of Green's conjecture for an even genus $g=2 k$ curve $C$ lying on a $K 3$ surface $X$. In this case, the determinantal variety is a Grassmannian $G(\mathrm{k}+2,2)$ produced out of a rank 2 Lazarsfeld-Mukai bundle on $X$ (itself resulting from a minimal pencil on $C$ ). Voisin then proves that the length of the linear strand of the resolution of $C$ is equal to that of $G(k+2,2)$. See also the recent paper $\left[\mathrm{AFP}^{+} 19\right]$, which removes the need to study Hilbert schemes as in Voisin's original approach.

For another example, a minimal pencil $f: C \rightarrow \mathbf{P}^{1}$ of degree $k$ induces a scroll

$$
X_{L}:=\bigcup_{p \in \mathbf{P}^{1}}\left\langle f^{-1}(p)\right\rangle \subseteq \mathbf{P}^{r(L)} .
$$

The scroll is a determinantal variety containing the embedded curve $(C, L)$. In this case, the Lascoux resolution of the scroll simplifies to an explicit resolution first found by Eagon-Northcott; see [Wey03, (6.1.6)]. By comparing the syzygies of $\left(C, \omega_{C}\right)$ to those of the scroll $X_{\omega_{C}}$, Schreyer has classified all Betti tables of canonical curves of genus $g \leqslant 9$ in geometric terms [Sch86].

If $\operatorname{deg}(L) \geqslant 2 g+k$, then the Eagon-Northcott complex shows $K_{p, 1}\left(X_{L}, \mathcal{O}(1)\right)=0$ for $p>$ $r(L)-k$, whereas $b_{r(L)-k, 1}\left(X_{L}, \mathcal{O}(1)\right)=r(L)-k$. Thus Theorem 1.1 can be interpreted as saying that all linear $(r(L)-k)$ th syzygies of the curve arise from the scroll; that is, restriction induces an isomorphism $K_{r(L)-k, 1}\left(X_{L}, \mathcal{O}(1)\right) \simeq K_{r(L)-k, 1}(C, L)$. Since there are no relations amongst the $(r(L)-k)$ th linear syzygies of the scroll $X_{L}$, the isomorphism above implies the vanishing $b_{p, 1}(C, L)=0$ for $p>r(L)-k$, as predicted by [EL15]. In this way, Theorem 1.1 provides a more 


\section{Projecting Syzygies OF CURVES}

geometric explanation for this vanishing statement, which was originally proved for line bundles of asymptotically high degree using Serre vanishing.

Theorem 1.1 also provides the first non-trivial step towards understanding the non-zero terms in the minimal free resolution of $C$, by giving the value of the last non-zero Betti number on the linear strand. This extremal Betti number is known to be responsible for much of the variance in the Betti tables of curves [Sch03].

The bound of Theorem 1.1 is optimal. Suppose $g=2 k-1$ and that $C$ is a general curve of genus $g$ and gonality $k$. Then $C$ admits both $A \in W_{k}^{1}(C)$ and $B \in W_{k+1}^{1}(C)$. Set $L:=\omega_{C} \otimes B$. There are two scrolls containing $C$, the scroll $X_{L}$ given by the union of $\operatorname{Span}(D)$ for $D \in|A|$ and the scroll $Y_{L}=\bigcup_{E \in|B|} \operatorname{Span}(E)$. Both scrolls contribute syzygies, implying $b_{r(L)-k, 1}(C, L)>$ $r(L)-k$.

The second goal of this paper is to use geometric constructions to refine the existing results on Green's conjecture for special canonical curves [Gre84a]. Firstly, we prove that Green's conjecture holds for general covers of elliptic curves of arbitrary degree, generalizing a result of AproduFarkas when $d=3$; see [AF12].

Theorem 1.2. Fix any elliptic curve $E$, and let $f: C \rightarrow E$ be a general degree $d$ primitive cover of $E$ for $d \geqslant 3$. Then Green's conjecture holds for $C$.

Our interest in this result derives from several angles. On the one hand, the strongest result known up to now on Green's conjecture is Aprodu's theorem [Apr05], stating that Green's conjecture holds for any $k$-gonal curve $C$ of genus $g$ provided that one has the linear growth condition

$$
\operatorname{dim} W_{k+n}^{1}(C) \leqslant n \quad \text { for all } 0 \leqslant n \leqslant g+2-2 k .
$$

Aprodu's theorem was a crucial part of Aprodu-Farkas' well-known result that Green's conjecture holds for curves on arbitrary K3 surfaces [AF11].

The linear growth condition is conjecturally equivalent to requiring

$$
\operatorname{dim} W_{k}^{1}(C)=0
$$

provided $g \leqslant 2 k-1$. Hence the most interesting open case of Green's conjecture is for curves where this condition fails, that is, for curves of non-maximal gonality with infinitely many minimal pencils. Perhaps the most natural example of such curves arises from general covers $f: C \rightarrow E$ of elliptic curves of degree $d$. Provided $2 d \leqslant\lfloor(g+3) / 2\rfloor$, such curves $C$ have gonality $2 d$. Note that we always have $\operatorname{dim} W_{2 d}^{1}(C) \geqslant 1$, by pulling back line bundles of degree 2 from $E$.

Covers of elliptic curves also play an important role in the computer experiments of Schreyer $[S c h 03, \S 6]$, which indicate that the Betti tables of covers of elliptic curves seem to behave very differently from general curves of gonality $2 d$. In particular, the last Betti number $b_{g-2 d, 1}\left(C, \omega_{C}\right)$ in the 2-linear strand need not be a multiple of $g-k$, which is rather strange given the computations of Hirschowitz-Ramanan [HR98]. It is this phenomenon that led us to consider such curves.

The last result we prove is an improvement to the prior results on Green's conjecture for curves of even genus and maximal gonality. Recall that results of Hirschowitz-Ramanan [HR98], when combined with [Voi05], establish Green's conjecture for all curves of odd genus and maximal gonality. In the case of curves of even genus $g=2 n$ and maximal gonality $k=n+1$, the best known prior result is Aprodu's theorem, establishing the conjecture for curves $C$ with $\operatorname{dim} W_{n+1}^{1}(C)=0$. One always has $\operatorname{dim} W_{n+1}^{1}(C) \leqslant 1$ by [FHL84], so it remains to analyse the 


\section{KEMENY}

case

$$
\operatorname{dim} W_{n+1}^{1}(C)=1 .
$$

We go quite a long way towards resolving this remaining case.

Theorem 1.3. Let $C$ be a smooth curve of genus $g=2 n$ and gonality $k=n+1$. Suppose that for $x, y \in C$ general, there is at most one $A \in W_{n+1}^{1}(C)$ such that $A(-x-y)$ is effective. Further assume $h^{0}\left(C, A^{\otimes 2}\right)=3$. Then $C$ satisfies Green's conjecture.

An example of a curve satisfying the assumptions above is given by a general degree $d$ primitive cover $f: C \rightarrow E$ of an elliptic curve, where $n$ is odd, $2 d=n+1$, and $C$ has genus $2 n=4 d-2$.

\subsection{Techniques}

The results above are proven by associating geometric varieties and constructions to syzygy spaces. In the process, we obtain some technical results on syzygies which we believe are of independent interest.

To explain the techniques, first recall that syzygy spaces $K_{i, j}(X, L)$ can be identified with the middle cohomology of

$$
\stackrel{i+1}{\bigwedge} H^{0}(L) \otimes H^{0}((j-1) L) \stackrel{\delta}{\rightarrow} \bigwedge^{i} H^{0}(L) \otimes H^{0}(j L) \stackrel{\delta}{\rightarrow} \bigwedge^{i-1} H^{0}(L) \otimes H^{0}((j+1) L),
$$

where $\delta$ is the Koszul differential.

Suppose that $V \subseteq H^{0}(X, L)$ is a codimension 1 subspace, and let $\operatorname{pr}_{V}: \mathbf{P}\left(H^{0}(X, L)^{\vee}\right) \rightarrow$ $\mathbf{P}\left(V^{\vee}\right)$ be the associated projection morphism. As first observed by Green [Gre84b, $\S 1$.b], it can be useful to compare the syzygies of $\Gamma_{X}(L)$ when considered as a $\operatorname{Sym}(V)$-module to its syzygies as a $\operatorname{Sym}\left(H^{0}(X, L)\right)$-module. Namely, letting $K_{i, j}\left(\Gamma_{X}(L), V\right)$ denote the middle cohomology of

$$
\stackrel{i+1}{\bigwedge} V \otimes H^{0}((j-1) L) \stackrel{\delta}{\rightarrow} \bigwedge^{i} V \otimes H^{0}(j L) \stackrel{\delta}{\rightarrow} \bigwedge^{i-1} V \otimes H^{0}((j+1) L),
$$

we have the projection map

$$
\operatorname{pr}_{V}: K_{i, j}(X, L) \rightarrow K_{i-1, j}\left(\Gamma_{X}(L), V\right)
$$

see [AN10, §2.2.1]. If we suppose that $\phi_{L}: X \hookrightarrow \mathbf{P}\left(H^{0}(X, L)^{\vee}\right)$ is projectively normal, that $x \in X \subseteq \mathbf{P}\left(H^{0}(X, L)^{\vee}\right)$ is a point corresponding to a codimension 1 subspace $V \subseteq H^{0}(X, L)$, and that further the projected variety $\operatorname{pr}_{x}(X) \subseteq \mathbf{P}\left(V^{\vee}\right)$ is projectively normal, then the projection map factors through a map

$$
\operatorname{pr}_{x}: K_{i, j}(X, L) \rightarrow K_{i-1, j}\left(\operatorname{pr}_{x}(X), \mathcal{O}_{\operatorname{pr}_{x}(X)}(1)\right),
$$

where we have an inclusion $K_{i-1, j}\left(\operatorname{pr}_{x}(X), \mathcal{O}_{\mathrm{pr}_{x}(X)}(1)\right) \subseteq K_{i-1, j}\left(\Gamma_{X}(L), V\right)$.

The projection map $\operatorname{pr}_{x}: K_{i, j}(X, L) \rightarrow K_{i-1, j}\left(\operatorname{pr}_{x}(X), \mathcal{O}_{\operatorname{pr}_{x}(X)}(1)\right)$ has been used by Choi, Kang, and Kwak to prove that if the projective variety $X \subseteq \mathbf{P}\left(H^{0}(X, L)^{\vee}\right)$ as above satisfies the Green-Lazarsfeld property $\left(N_{p}\right)$ and if, furthermore, $\operatorname{pr}_{x}(X) \simeq X$, then $\operatorname{pr}_{x}(X) \subseteq \mathbf{P}\left(V^{\vee}\right)$ satisfies property $\left(N_{p-1}\right)$; see [CKK06]. Specializing to the case where $X$ is a smooth curve $C$, Aprodu has used the projection map to study the Green and Green-Lazarsfeld conjectures for curves [Apr02].

Our first technical contribution is to relate the projection map to Green's notion of syzygy scheme. For a syzygy $\alpha \neq 0 \in K_{p, 1}(X, L)$, the syzygy scheme $\operatorname{Syz}(\alpha)$ is defined to be the 


\section{Projecting Syzygies OF CURVES}

largest variety $Y \subseteq \mathbf{P}\left(H^{0}(X, L)^{\vee}\right)$ containing $X$ such that $\alpha$ arises via restriction from a syzygy $\alpha^{\prime} \in K_{p, 1}\left(Y, \mathcal{O}_{Y}(1)\right)$; see [Gre82]. It is well known that one has the containment

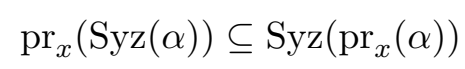

for any $x \in X$, relating the syzygy scheme of a syzygy to the syzygy scheme of its projection; see [AN10, §3]. Furthermore, it is known that if $Z \subseteq X$ is a spanning set of $\mathbf{P}\left(H^{0}(X, L)^{\vee}\right)$ and $\alpha \neq 0$, then there is some $x \in Z$ such that $\operatorname{pr}_{x}(\alpha) \neq 0$ or, equivalently, $\operatorname{Syz}\left(\operatorname{pr}_{x}(\alpha)\right) \neq \mathbf{P}\left(V^{\vee}\right)$; see [AN10, Proposition 2.14].

We provide here a generalization of both of the above statements, showing that one can completely recover the syzygy scheme of an element $\alpha \in K_{p, 1}(X, L)$ from the syzygy scheme of projections $\operatorname{pr}_{x}(\alpha)$. Let $X \subseteq \mathbf{P}\left(H^{0}(X, L)^{\vee}\right)$ be an integral projective variety embedded by a very ample line bundle $L$ as above, and assume for simplicity that $X$ is projectively normal. For any $x \in X \subseteq \mathbf{P}\left(H^{0}(X, L)^{\vee}\right)$, let $W_{x} \subseteq H^{0}(X, L)$ be the corresponding codimension 1 subspace.

Theorem 1.4. Let $X$ be as above, and let $Z \subseteq X$ be a subset spanning $\boldsymbol{P}\left(H^{0}(X, L)^{\vee}\right)$. Assume that for all $x \in Z$, the variety $\operatorname{pr}_{x}(X) \subseteq \boldsymbol{P}\left(W_{x}^{\vee}\right)$ is linearly normal and non-degenerate. Let $\alpha \neq 0 \in K_{p, 1}(X, L)$. Then

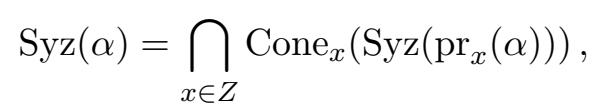

where, for any $Y \in \boldsymbol{P}\left(W_{x}^{\vee}\right)$, the variety $\operatorname{Cone}_{x}(Y) \subseteq \boldsymbol{P}\left(V^{\vee}\right)$ is the cone with vertex $x$.

Our next technical result is related to the following early application of the technique of projection of syzygies.

THEOREM 1.5 (Aprodu's projection theorem). Let $C$ be a smooth curve of genus $g$, and suppose that $x, y \in C$ are distinct points. Let $D$ be the $(g+1)$-nodal curve obtained by identifying $x$ and $y$. Suppose $K_{p, 1}\left(C, \omega_{C}\right)=0$. Then $K_{p+1,1}\left(D, \omega_{D}\right)=0$.

This allows one to prove the generic Green's conjecture for curves of a fixed gonality by induction on the genus. Perhaps the most interesting case of Theorem 1.5 is when $C$ is a curve of gonality $k$ and Clifford index $k-2$, with minimal pencil $f: C \rightarrow \mathbf{P}^{1}$ of degree $k$, and where $p=g-k+1$ and $x, y \in C$ are distinct points with $f(x)=f(y)$. In this case, Theorem 1.5 implies that if $C$ satisfies Green's conjecture, then the nodal curve $D$ obtained by identifying $x$ and $y$ also has gonality $k$ and Clifford index $k-2$, and furthermore $D$ satisfies Green's conjecture. This provides an approach to proving Green's conjecture for general curves of a fixed gonality using induction and was one of Aprodu's main motivations for formulating Theorem 1.5.

It is natural to ask for a converse of this result, that is, to find an assumption on $D$ as above to guarantee that $C$ satisfies Green's conjecture. We recall from [SSW13, FK19, BS18] that Schreyer has stated the following strengthening of Green's conjecture.

Conjecture 1.6 (Schreyer's conjecture). Let $C$ be a curve of genus $g$ and non-maximal gonality $3 \leqslant k \leqslant(g+1) / 2$. Assume that $W_{k}^{1}(C)=\{A\}$ is a reduced single point and $A$ is the unique line bundle of degree at most $g-1$ achieving the Clifford index. Then

$$
b_{g-k, 1}\left(C, \omega_{C}\right)=g-k .
$$

Note that the condition that $W_{k}^{1}(C)$ is reduced is equivalent to demanding $h^{0}\left(C, A^{\otimes 2}\right)=3$.

Schreyer's conjecture has been proven under the "bpf-linear growth" genericity assumption in [FK19]. As explained earlier, Schreyer's conjecture implies Green's conjecture

$$
b_{g-k+1,1}\left(C, \omega_{C}\right)=0
$$




\section{KEMENY}

and, further, implies that all syzygies at the end of the 2-linear strand of the canonical curve come from the scroll $X_{\omega_{C}}$. In terms of syzygy schemes, Schreyer's conjecture is equivalent to the statement that $\operatorname{Syz}(\alpha)=X_{\omega_{C}}$ for all $\alpha \neq 0 \in K_{g-k, 1}\left(C, \omega_{C}\right)$.

Our partial converse to the Aprodu projection theorem then reads as follows.

THEOREM 1.7. Let $D$ be the 1-nodal $k$-gonal curve as above, with normalization the smooth curve $C$ of genus $g$ and line bundle $B \in W_{k}^{1}(D)$ satisfying $\nu^{*} B \simeq A$. Assume

(i) $h^{0}\left(C, A^{\otimes 2}\right)=3$ and

(ii) $b_{g+1-k, 1}\left(D, \omega_{D}\right)=g+1-k$.

Then $K_{g+1-k, 1}\left(C, \omega_{C}\right)=0$.

In other words, if the $k$-gonal nodal curve $D$ of genus $g+1$ satisfies Schreyer's conjecture, then the smooth $k$-gonal curve $C$ of genus $g$ satisfies Green's conjecture.

We now briefly explain how these technical results imply the main results. The key ingredients in the proof of Theorem 1.1 are Theorem 1.4 together with the following important result of Eisenbud-Popescu [EP99].

Theorem 1.8 (Eisenbud-Popescu). Let $X \subseteq \boldsymbol{P}^{r}$ be a rational normal scroll of degree $f$ and $0 \neq \alpha \in K_{f-1,1}\left(X, \mathcal{O}_{X}(1)\right)$. Then $\operatorname{Syz}(\alpha)=X$.

Theorem 1.2 on Green's conjecture for elliptic curves is proved by combining the Aprodu projection theorem with an analysis of the Brill-Noether theory of covers $f: C \rightarrow E$ as above, along the lines of [AC81]. Lastly, Theorem 1.3 on Green's conjecture for curves of even genus is an immediate corollary of Theorem 1.7.

\section{Projections of syzygy schemes}

The goal of this section is to prove a precise relationship between the syzygy scheme of a linear syzygy and that of its projection. We first need to recall the notion of a syzygy scheme. Let $X \subseteq \mathbf{P}^{n}$ be a closed subscheme such that $X$ is non-degenerate; that is, the restriction map

$$
V:=H^{0}\left(\mathbf{P}^{n}, \mathcal{O}_{\mathbf{P}^{n}}(1)\right) \rightarrow H^{0}\left(X, \mathcal{O}_{X}(1)\right)
$$

is injective. We let $S(X)$ denote the homogeneous coordinate ring and let $K_{p, q}(S(X), V)$ denote the syzygies of $S(X)$ as a $\operatorname{Sym}(V)=S\left(\mathbf{P}^{n}\right)$-module. We have an isomorphism

$$
K_{p, 1}(S(X), V) \simeq K_{p-1,2}\left(I_{X}, V\right),
$$

where $I_{X}$ is the ideal of $X$, by [AN10, Proposition 1.27], and further

$$
K_{p-1,2}\left(I_{X}, V\right)=\operatorname{Ker}\left(\bigwedge^{p-1} V \otimes\left(I_{X}\right)_{2} \rightarrow \bigwedge^{p-2} V \otimes\left(I_{X}\right)_{3}\right) .
$$

If $Y \subseteq \mathbf{P}^{n}$ is any closed subscheme containing $X$, then the inclusion $I_{Y} \subseteq I_{X}$ induces an inclusion

$$
K_{p, 1}(S(Y), V) \simeq K_{p-1,2}\left(I_{Y}, V\right) \stackrel{\operatorname{res}_{X}^{Y}}{\longrightarrow} K_{p-1,2}\left(I_{X}, V\right) \simeq K_{p, 1}(S(X), V) .
$$

Definition 2.1 ([AN10, $\S 3]$ ). Let $\alpha$ be a non-zero element of $K_{p, 1}(S(X), V)$. Then the syzygy scheme $\operatorname{Syz}(\alpha) \subseteq \mathbf{P}^{n}$ is defined to be the largest closed subscheme $Y \subseteq \mathbf{P}^{n}$ containing $X$ such that $\alpha \in \operatorname{Im}\left(\operatorname{res}_{X}^{Y}\right)$. 


\section{ProjeCting SYZYGIES OF CURVES}

We recall some basic facts about syzygy schemes from $[\mathrm{AN} 10, \S 3]$. Let $V$ be a vector space of dimension $n+1$, and identify $\mathbf{P}^{n} \simeq \mathbf{P}\left(V^{\vee}\right):=\operatorname{Proj}(\operatorname{Sym}(V))$. For any $x \in V^{\vee}$, let $W_{x} \subseteq V$ denote the kernel of $x: V \rightarrow \mathbb{C}$, and let $i_{x}: \bigwedge^{p} V \rightarrow \bigwedge^{p-1} W_{x}$ be the contraction mapping defined by

$$
i_{x}\left(v_{1} \wedge \cdots \wedge v_{p}\right)=\sum_{i}(-1)^{i} v_{1} \wedge \cdots \wedge \hat{v}_{i} \wedge \cdots \wedge v_{p} \otimes x\left(v_{i}\right)
$$

The following statement is [AN10, Lemma 3.7 and Proposition 3.15].

Proposition 2.2. Let $X \subseteq \boldsymbol{P}\left(V^{\vee}\right)$ be a non-degenerate linearly normal projective variety.

(i) Let $\alpha \in K_{p, 1}(S(X), V)$, and let $\bar{\alpha} \in \operatorname{Hom}\left(V^{\vee}, \bigwedge^{p} V\right) \simeq \bigwedge^{p} V \otimes V$ be a representative for $\alpha$. Then $\operatorname{Syz}(\alpha)=\left\{[x] \in \boldsymbol{P}\left(V^{\vee}\right) \mid i_{x}(\bar{\alpha}(x))=0\right\}$.

(ii) For any $x \in V^{\vee}$, let $\operatorname{pr}_{x}: \boldsymbol{P}\left(V^{\vee}\right) \rightarrow \boldsymbol{P}\left(W_{x}^{\vee}\right)$ denote the projection with centre $x$. Suppose that both $X$ and $Y:=\operatorname{pr}_{x}(X)$ are linearly normal as well as non-degenerate. Then for any non-zero $\alpha \in K_{p, 1}(S(X), V)$, we have $x \in \operatorname{Syz}(\alpha)$ if and only if there exists a $\beta: W_{x}^{\vee} \rightarrow$ $\bigwedge^{p-1} W_{x}$ such that we have a commutative diagram

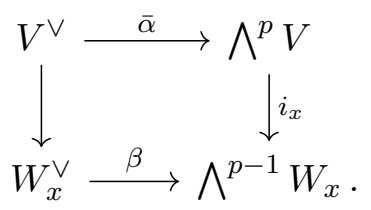

Part (ii) of Proposition 2.2 can be rephrased. In the notation of the proposition, assume that $X$ and $Y$ are linearly normal and non-degenerate. Recall from [AN10, $\S 2.2 .1$ that there is a "projection map"

$$
\operatorname{pr}_{x}: K_{p, 1}(S(X), V) \rightarrow K_{p-1,1}\left(S(X), W_{x}\right) .
$$

Then one can rephrase Proposition 2.2(ii) as $x \in \operatorname{Syz}(\alpha)$ if and only if $\operatorname{pr}_{x}(\alpha) \in K_{p-1,1}\left(S(Y), W_{x}\right)$.

The next result provides an improvement of [AN10, Lemma 3.17(ii)].

Theorem 2.3. Let $X \subseteq \boldsymbol{P}\left(V^{\vee}\right)$ be an integral projective variety, and let $Z \subseteq X$ be a set such that $\operatorname{Span}(Z)=\boldsymbol{P}\left(V^{\vee}\right)$. Assume that for all $x \in Z$, both $X$ and $\operatorname{pr}_{x}(X) \subseteq \boldsymbol{P}\left(W_{x}^{\vee}\right)$ are linearly normal and non-degenerate. Let $\alpha \neq 0 \in K_{p, 1}(S(X), V)$. Then

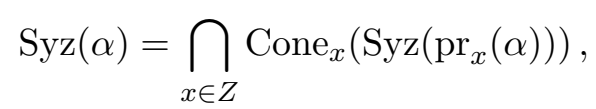

where, for any $Y \in \boldsymbol{P}\left(W_{x}^{\vee}\right)$, the variety $\operatorname{Cone}_{x}(Y) \subseteq \boldsymbol{P}\left(V^{\vee}\right)$ is the cone with vertex $x$.

Proof. Let $x \in Z \subseteq \operatorname{Syz}(\alpha)$. By Proposition 2.2(ii), for any $y \in V^{\vee}$, we have a commutative diagram

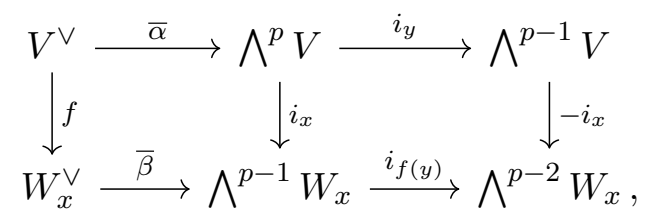

where $\bar{\alpha}$ and $\bar{\beta}$ represent $\alpha$ and $\operatorname{pr}_{x}(\alpha)$, respectively, and $f:=\operatorname{pr}_{x}$ is the usual projection map of vector spaces. By Proposition 2.2(i), we have $y \in \operatorname{Syz}(\alpha)$ if and only if $i_{y}(\bar{\alpha}(y))=0$. Hence if $y \in \operatorname{Syz}(\alpha)$, then $i_{f(y)}(\bar{\beta}(f(y)))=0$, and hence $\operatorname{pr}_{x}(y)=f(y) \in \operatorname{Syz}\left(\operatorname{pr}_{x}(\alpha)\right)$, by Proposition 2.2 


\section{KEMENY}

once again. Thus $y \in \operatorname{Cone}\left(\operatorname{Syz}\left(\operatorname{pr}_{x}(\alpha)\right)\right)$. Hence we have established the inclusion

$$
\operatorname{Syz}(\alpha) \subseteq \bigcap_{x \in Z} \operatorname{Cone}_{x}\left(\operatorname{Syz}_{\left.\left(\operatorname{pr}_{x}(\alpha)\right)\right)} .\right.
$$

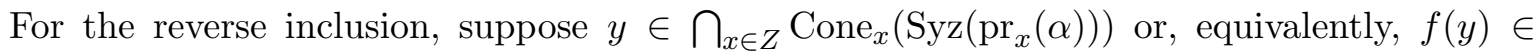
$\operatorname{Syz}\left(\operatorname{pr}_{x}(\alpha)\right)$ for all $x \in Z$. Then

$$
i_{x}\left(i_{y}(\bar{\alpha}(y))\right)=0 \quad \text { for all } x \in Z .
$$

By Proposition 2.2, we need to show $i_{y}(\bar{\alpha}(y))=0$. Since $Z$ spans $\mathbf{P}\left(V^{\vee}\right)$, it suffices to observe that for any non-zero $u \in \bigwedge^{p-1} V$,

(1) there is some $z \in V^{\vee}$ such that $i_{z}(u) \neq 0$;

(2) the set of $z \in V^{\vee}$ such that $i_{z}(u)=0$ forms a subspace.

Indeed, statement (2) is obvious, whereas for statement (1), we observe that $i_{z}(u)=0$ if and only if $u \in \bigwedge^{p-1} W_{z}$ by [AN10, Remark 1.3]. Hence, statement (1) follows from the trivial observation that there exists a codimension 1 subspace $W \subseteq V$ with $u \notin \bigwedge^{p-1} W$.

\section{Extremal syzygies of embedded curves}

In this section, we will prove [FK19, Conjecture 0.5], which states that all extremal syzygies of a general curve of non-maximal gonality embedded by a complete linear system of sufficiently high degree arise from a scroll. Let $C$ be a smooth $k$-gonal curve of genus $g \geqslant 2 k-1$, for $k \geqslant 2$, and $L \in \operatorname{Pic}(C)$ a line bundle. If $C$ is sufficiently general, then there exists a unique line bundle $A$ with $\operatorname{deg}(A)=k$ and $h^{0}(A) \geqslant 2$; see [AC81]. Further, for such a general $k$-gonal curve, $h^{0}\left(A^{2}\right)=3$ or, equivalently, the Brill-Noether locus $W_{k}^{1}(C)$ is smooth. Assume further $h^{1}(L-A)=0$. Consider the embedding $\phi_{L}: C \hookrightarrow \mathbf{P}^{r(L)}$ for $r(L):=h^{0}(L)-1$ and the scroll [Sch86]

$$
X_{L}:=\bigcup_{D \in|A|} \operatorname{Span}(D) \subseteq \mathbf{P}^{r(L)} .
$$

The scroll $X_{L}$ has degree $r(L)+1-k$ in $\mathbf{P}^{r(L)}$ and has Betti numbers

$$
b_{p, 1}\left(X_{L}, \mathcal{O}_{X_{L}}(1)\right)=p\left(\begin{array}{c}
r(L)+1-k \\
p+1
\end{array}\right)
$$

whereas $b_{p, q}\left(X_{L}, \mathcal{O}_{X_{L}}(1)\right)=0$ for $q \geqslant 2$. As seen in the previous section, we have an inclusion

$$
\operatorname{res}_{C}: K_{p, 1}\left(X_{L}, \mathcal{O}_{X_{L}}(1)\right) \hookrightarrow K_{p, 1}(C, L) .
$$

Conjecture 0.6 from [FK19] states that if $\operatorname{deg}(L) \geqslant 2 g+k$, then $\operatorname{res}_{C}$ is surjective in the extremal case $p=r(L)-k$, which is the largest value of $p$ such that $b_{p, 1}\left(X_{L}, \mathcal{O}_{X_{L}}(1)\right) \neq 0$. Note that the surjectivity of $\operatorname{res}_{C}: K_{r(L)-k, 1}\left(X_{L}, \mathcal{O}_{X_{L}}(1)\right) \hookrightarrow K_{r(L)-k, 1}(C, L)$ is equivalent to the statement

$$
X_{L} \subseteq \operatorname{Syz}(\alpha) \text { for all } \alpha \in K_{r(L)-k, 1}(C, L) .
$$

We will prove equation (3.1) using Proposition 2.3 together with the following important result of Eisenbud-Popescu.

Theorem 3.1 ([EP99]). Let $X \subseteq \boldsymbol{P}^{r}$ be a rational normal scroll of degree $f$ and $0 \neq \alpha \in$ $K_{f-1,1}\left(X, \mathcal{O}_{X}(1)\right)$. Then $\operatorname{Syz}(\alpha)=X$.

The next proposition will be the key step in our proof. 


\section{ProjeCting SYZYGIES OF CURVES}

Proposition 3.2. Let $C$ be a general $k$-gonal curve of genus $g \geqslant 2 k-1$ and $k \geqslant 2$. Suppose $b_{r(L)-k, 1}(C, L)=r(L)-k$ for some line bundle $L$ with $h^{1}(L-A)=0$. Then $b_{r(L)+1-k, 1}(C, L(x))$ $=r(L)+1-k$ for any $x \in C$.

Proof. Set $M=L(x)$, and let $\alpha \in K_{r(M)-k, 1}(C, M)$ be non-zero. By assumption, the Betti number $b_{r(M(-x))-k, 1}(C, M(-x))$ takes the minimal possible value $r(M(-x))-k$. Hence, by the semicontinuity of Koszul cohomology, there exists a dense open $U \subseteq C$ such that we have $b_{r(M(-y))-k, 1}(C, M(-y))=r(M(-y))-k$ for all $y \in C$ and, further, $\operatorname{pr}_{y}(\alpha) \neq 0$ by [AN10, Proposition 2.14]. By Proposition 2.3,

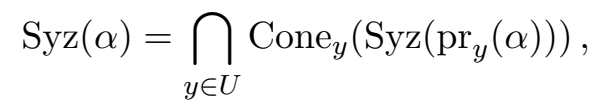

where $\operatorname{pr}_{y}(\alpha) \in K_{r(M(-y))-k, 1}(C, M(-y))$. By assumption, the map

$$
\operatorname{res}_{C}: K_{r(M(-y))-k, 1}\left(X_{M(-y)}, \mathcal{O}_{M(-y)}(1)\right) \rightarrow K_{r(M(-y))-k, 1}(C, M(-y))
$$

is an isomorphism, so Theorem 3.1 gives $\operatorname{Syz}\left(\operatorname{pr}_{y}(\alpha)\right)=X_{M(-y)} \subseteq \mathbf{P}^{r(M)-1}$. Observe that $X_{M(-y)}=\operatorname{pr}_{y}\left(X_{M}\right)$. Hence $X_{M} \subseteq$ Cone $_{y}\left(\operatorname{pr}_{y}\left(X_{M(-y)}\right)\right)$. Hence $X_{M} \subseteq \operatorname{Syz}(\alpha)$, as required.

We will prove equation (3.1) by induction. The initial step is provided in the following theorem.

Theorem 3.3. Let $g=2 i+1$, and let $C$ be a smooth curve of genus $g$ and gonality $i+1$. Assume that there is a unique $A \in W_{i+1}^{1}(C)$ and that, further, for such a line bundle $A$, we have $h^{0}\left(A^{2}\right)=3$. Let $L$ be a line bundle of degree $2 g$ which is $i$-very ample; equivalently, $L-K_{C}$ is not in the difference variety $C_{i+1}-C_{i-1}$. Then $b_{i, 1}(C, L)=i$.

Proof. This follows from the results in [FK16], in particular the equality of cycles

$$
\mathfrak{S} \mathfrak{y} \mathfrak{z}=\mathfrak{S e c}+i \mathfrak{h} \mathfrak{u} \mathfrak{r}
$$

on $\mathcal{M}_{g, 2 g}$. Namely, if $D=\sum_{i=1}^{2 g} x_{i} \in|L|$ is a reduced divisor, then the marked curve $(C, D) \in$ $\mathcal{M}_{g, 2 g}$ is not in the divisor $\mathfrak{S e c}$ by definition. Hence on an appropriate étale cover $\phi: S \rightarrow \mathcal{M}_{g, 2 g}$ about $p=[C, D]$, the order of vanishing of the function defining the divisor $\mathfrak{S} \mathfrak{y} \mathfrak{z}(\phi)$ at $p$ is given by $i$ multiplied by the order of vanishing of $\mathfrak{h u r}(\phi)$. But the assumption that there is a unique $A \in W_{i+1}^{1}(C)$ and $h^{0}\left(A^{2}\right)=3$ shows that $\mathfrak{h u r}(\phi)$ vanishes to first order at $p$, and thus $\mathfrak{S} \mathfrak{y} \mathfrak{z}(\phi)$ vanishes to order $i$, so that $b_{i, 1}(C, L) \leqslant i$ by the construction of the syzygy divisor $\mathfrak{S} \mathfrak{y} ;$; cf. [FK19, Theorem 3.1]. The condition $L-K_{C} \notin C_{i+1}-C_{i-1}$ implies $h^{1}(L-A)=0$ and that the scroll $X_{L}$ has degree $i+1$. As we have already seen that $b_{i, 1}(C, L) \geqslant b_{i, 1}\left(X_{L}, \mathcal{O}_{X_{L}}(1)\right)=i$, this completes the proof.

As an immediately corollary, we can prove [FK19, Conjecture 0.6] for any smooth curve $C$ of odd genus $g \geqslant 5$ and submaximal gonality $k=(g+1) / 2$, assuming that there is a unique $A \in W_{i+1}^{1}(C)$ and, further, $h^{0}\left(A^{2}\right)=3$.

Corollary 3.4. Let $C$ be a smooth curve of odd genus $g=2 i+1$ for $i \geqslant 3$ and submaximal gonality $i+1$. Assume that there is a unique $A \in W_{i+1}^{1}(C)$ and, further, $h^{0}\left(A^{2}\right)=3$. Then $b_{r(L)-i-1,1}(C, L)=r(L)-i-1$ for all line bundles of degree $\operatorname{deg}(L) \geqslant 2 g+i+1$.

Proof. By Proposition 3.2, it suffices to assume $\operatorname{deg}(L)=2 g+i+1=5 i+3$. By Theorem 3.3 together with Proposition 3.2, it suffices to show that there is an effective divisor $D \in C_{i+1}$ such that $L-K_{C}-D \notin C_{i+1}-C_{i-1}$. By [FK19, proof of Theorem 0.2], it suffices to show that the secant 


\section{KEMENY}

variety $V_{2 i+2}^{2 i+1}(L)$ has dimension at most $i$, and for this it is sufficient to show $W_{i+3}^{2}(C)=\emptyset$. In the range $i \geqslant 3$, any $B \in W_{i+3}^{2}(C)$ would contribute to the Clifford index, so it suffices to show that $\operatorname{Cliff}(C)=i-1$ and that, further, $A$ is the unique line bundle of degree at most $g-1$ achieving the Clifford index. But this follows from the well-known result of Hirschowitz-Ramanan that our assumptions imply that Schreyer's conjecture holds for $C$, see [HR98] and [FK19, Theorem 3.1], together with the easy direction of Schreyer's conjecture [SSW13, Proposition 4.10].

We now arrive at the main result of this section.

Theorem 3.5. Let $C$ be a general $k$-gonal curve $C$ of genus $g \geqslant 2 k-1$ for $k \geqslant 4$. Let $L$ be an arbitrary line bundle on $C$ of degree $\operatorname{deg}(L) \geqslant 2 g+k$. Then $b_{r(L)-k, 1}(C, L)=r(L)-k$.

Proof. We mirror the proof of [FK19, Theorem 0.1]. Namely, fix $k \geqslant 4$. We prove the result by induction on the genus $g$ of $C$. If $g=2 k-1$, then the claim is Corollary 3.4. So, assume that the claim holds for a general $k$-gonal curve $C$. By Proposition 3.2, it suffices to show that for a general $k$-gonal curve $X^{\prime}$ of genus $g+1$ and any line bundle $L^{\prime}$ on $X^{\prime}$ of degree $2 g+2+k$, we have $b_{g+1,1}\left(X^{\prime}, L^{\prime}\right)=g+1$. Using Proposition 3.2 once more, it is further sufficient to show that there exists a point $p \in X^{\prime}$ such that $b_{g, 1}\left(X^{\prime}, L^{\prime}(-p)\right)$ takes the lowest possible value $g$. Now let $X$ be the nodal curve $C \cup_{q} E$, where $C$ is a general $k$-gonal curve as above, $q$ is a branch point of some pencil $f: C \rightarrow \mathbf{P}^{1}$ of degree $k$, and $E$ is an elliptic curve. Then $X$ is a stable genus $g+1$ curve of compact type, which is a limit of smooth $k$-gonal curves. By the semicontinuity of Koszul cohomology, and since $X$ is of compact type, it suffices to show that for any line bundle $L$ on $X$ with

$$
\operatorname{deg}\left(L_{E}\right)=1, \quad \operatorname{deg}\left(L_{C}\right)=2 g+1+k,
$$

there exists a point $p^{\prime} \in E \backslash\{q\}$ with

(1) $b_{g, 1}\left(X, L\left(-p^{\prime}\right)\right)=g$,

(2) $h^{1}\left(X, L\left(-p^{\prime}\right)\right)=h^{1}\left(X, L^{\otimes 2}\left(-2 p^{\prime}\right)\right)=0$;

see [FK19, proof of Theorem 0.1]. Choose a general point $p^{\prime} \in E \backslash\{q\}$. Statement (2) follows immediately from the Mayer-Vietoris sequence

$$
0 \rightarrow L_{C}(-q) \rightarrow L\left(-p^{\prime}\right) \rightarrow L_{E}\left(-p^{\prime}\right) \rightarrow 0
$$

For statement (1), consider the commutative diagram

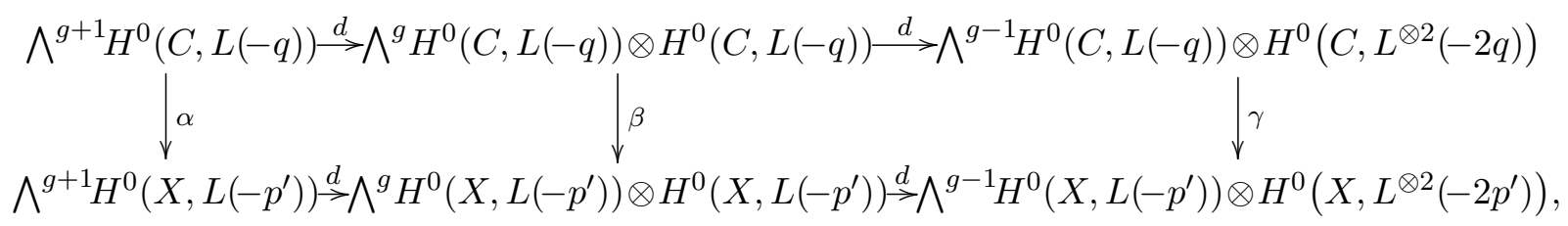

where $\alpha$ and $\beta$ are isomorphisms and $\gamma$ is induced from the natural composition

$$
H^{0}\left(C, L^{\otimes 2}(-2 q)\right) \hookrightarrow H^{0}\left(C, L^{\otimes 2}(-q)\right) \cong H^{0}\left(X, L^{\otimes 2}\left(-2 p^{\prime}\right)\right) .
$$

Since $\gamma$ is injective, we have a natural isomorphism

$$
K_{g, 1}(C, L(-q)) \stackrel{\sim}{\rightarrow} K_{g, 1}\left(X, L\left(-p^{\prime}\right)\right)
$$

induced on the cohomology of the rows in the above diagram. By the induction hypothesis, $b_{g, 1}(C, L(-q))=g$, which completes the proof. 


\section{ProjeCting SYZYGIES OF CURVES}

\section{A converse to Aprodu's projection theorem}

Recall the following theorem of Aprodu [Apr02].

THEOREM 4.1 (Aprodu's projection theorem). Let $C$ be a smooth curve of genus $g$, and suppose that $x, y \in C$ are distinct points. Let $D$ be the $(g+1)$-nodal curve obtained by identifying $x$ and $y$. Suppose $K_{p, 1}\left(C, \omega_{C}\right)=0$. Then $K_{p+1,1}\left(D, \omega_{D}\right)=0$.

One important application of this result is that it provides an approach to proving Green's conjecture for curves of a fixed gonality by induction on the genus. In this sense, the most interesting case of the Aprodu projection theorem is that where $p=g-k+1$, where $k$ is the gonality of $C$. The goal of this section is to prove a partial converse of Aprodu's theorem, allowing one to deduce Green's conjecture $K_{g-k+1,1}\left(C, \omega_{C}\right)=0$ by assuming that $D$ satisfies Schreyer's conjecture on syzygies arising from scrolls, which is a stronger assumption than Green's conjecture; see [SSW13, FK19].

Let $C$ be a smooth curve of genus $g$ and gonality $k \geqslant 3$, let $A \in W_{k}^{1}(C)$, and let $T \in|A|$ be a general divisor. Choose distinct points $x, y \in T$, and let $D$ be the nodal curve of genus $g+1$ obtained by identifying $x$ and $y$. Then there is a base-point-free line bundle $B$ on $D$ with two sections such that $\nu^{*} B \simeq A$, where $\nu: C \rightarrow D$ is the normalization morphism.

Embed $D$ in $\mathbf{P}^{g}$ via the canonical linear system, and let $\pi_{p}: \mathbf{P}^{g} \rightarrow \mathbf{P}^{g-1}$ be the projection from the node $p \in D$. Then the canonical curve $C \subseteq \mathbf{P}^{g-1}$ is the projection $\pi_{p}(D)$. Further, let $Z \subseteq \mathbf{P}^{g}$ denote the cone over $\pi_{p}(D)$ with vertex at $p$. Then $D \subseteq Z$. We denote by $\widetilde{\nu}: \widetilde{Z} \rightarrow Z$ the desingularization of $Z$. The strict transform $D^{\prime}$ of $D$ is isomorphic to $C$, and $\widetilde{\nu}_{D_{D^{\prime}}} \simeq \nu$.

By $[\operatorname{Har} 77, \S \mathrm{V} .2]$, we have $\widetilde{Z} \simeq \mathbf{P}\left(\mathcal{O}_{C} \oplus \omega_{C}\right)$ and $\operatorname{Pic}(\widetilde{Z}) \simeq \mathbb{Z}[\mathcal{H}] \oplus \iota^{*} \operatorname{Pic}(C)$, where $\mathcal{H}$ denotes the pull-back of the hyperplane section of $\mathbf{P}^{g}$ and

$$
\iota: \mathbf{P}\left(\mathcal{O}_{C} \oplus \omega_{C}\right) \rightarrow C
$$

is the projection map.

Lemma 4.2. We have $\mathcal{O}_{\widetilde{Z}}\left(D^{\prime}\right) \simeq \mathcal{O}_{\widetilde{Z}}(\mathcal{H}) \otimes \iota^{*} \mathcal{O}_{C}(x+y)$.

Proof. The strict transform $D^{\prime}$ corresponds to a section $s: C \rightarrow \mathbf{P}\left(\mathcal{O}_{C} \oplus \omega_{C}\right)$. We have $s^{*} \mathcal{H} \simeq$ $\nu^{*} \omega_{D} \simeq \omega_{C}(x+y)$. By [Har77, V.2, Proposition 2.6], we have a short exact sequence

$$
0 \rightarrow \mathcal{N} \rightarrow \mathcal{O}_{C} \oplus \omega_{C} \rightarrow s^{*} \mathcal{H} \rightarrow 0
$$

with $\iota^{*} \mathcal{N} \simeq \mathcal{H}\left(-D^{\prime}\right)$. By taking determinants of the short exact sequence above, we obtain

$$
\mathcal{N} \simeq \omega_{C}\left(-s^{*} \mathcal{H}\right) \simeq \mathcal{O}_{C}(-x-y) .
$$

Applying $\iota^{*}$ gives $\mathcal{O}_{\widetilde{Z}}\left(D^{\prime}\right) \simeq \mathcal{O}_{\widetilde{Z}}(\mathcal{H}) \otimes \iota^{*} \mathcal{O}_{C}(x+y)$, as claimed.

By Lemma 4.2, we have a short exact sequence

$$
0 \rightarrow \mathcal{H}^{\vee}\left(-\iota^{*}(x+y)\right) \rightarrow \mathcal{O}_{\widetilde{Z}} \rightarrow \mathcal{O}_{D^{\prime}} \rightarrow 0
$$

which induces an exact sequence of $\mathcal{S}$-modules, where $\mathcal{S}:=\operatorname{Sym}\left(H^{0}(\widetilde{Z}, \mathcal{H})\right)$,

$$
0 \rightarrow \bigoplus_{q \in \mathbb{Z}} H^{0}\left(\mathcal{H}^{\otimes(q-1)}\left(-\iota^{*}(x+y)\right)\right) \rightarrow \bigoplus_{q \in \mathbb{Z}} H^{0}\left(\mathcal{H}^{\otimes q}\right) \stackrel{\alpha}{\rightarrow} \bigoplus_{q \in \mathbb{Z}} H^{0}\left(C, \omega_{C}^{\otimes q}(q x+q y)\right),
$$

where we used the identification $C \simeq D^{\prime}$. We let

$$
\mathbb{M}:=\operatorname{Im}(\alpha)
$$




\section{KemenY}

be the image of $\alpha$. Then we have a short exact sequence of $\mathcal{S}$-modules

$$
0 \rightarrow \bigoplus_{q \in \mathbb{Z}} H^{0}\left(\mathcal{H}^{\otimes(q-1)}\left(-\iota^{*}(x+y)\right)\right) \rightarrow \bigoplus_{q \in \mathbb{Z}} H^{0}\left(\mathcal{H}^{\otimes q}\right) \rightarrow \mathbb{M} \rightarrow 0
$$

Proposition 4.3. The following statements hold:

(i) The inclusion $\mathbb{M} \subseteq \bigoplus_{q \in \mathbb{Z}} H^{0}\left(C, \omega_{C}^{\otimes q}(q x+q y)\right)$ induces an isomorphism $K_{p, 1}(\mathbb{M}, \mathcal{S}) \simeq$ $K_{p, 1}\left(C, \omega_{C}(x+y)\right)$ of syzygy spaces for all integers $p$.

(ii) Restricting to the hyperplane section induces an isomorphism $K_{p, q}(\widetilde{Z}, \mathcal{H}) \simeq K_{p, q}\left(C, \omega_{C}\right)$ for all $p, q$.

(iii) We have a natural exact sequence

$$
0 \rightarrow K_{p, 1}\left(C, \omega_{C}\right) \rightarrow K_{p, 1}\left(C, \omega_{C}(x+y)\right) \stackrel{\delta}{\rightarrow} K_{p-1,1}\left(\widetilde{Z},-\iota^{*}(x+y) ; \mathcal{H}\right) \rightarrow K_{p-1,2}\left(C, \omega_{C}\right)
$$

for all $p \in \mathbb{Z}$.

Proof. We have the isomorphism $H^{0}(\widetilde{Z}, \mathcal{H}) \simeq H^{0}\left(\mathcal{O}_{Z}(1)\right) \rightarrow H^{0}\left(\mathcal{H}_{D^{\prime}}\right) \simeq H^{0}\left(\mathcal{O}_{D}(1)\right)$, and thus the degree 1 piece $\mathbb{M}_{1}$ is isomorphic to $\left.H^{0}\left(C, \omega_{C}(x+y)\right)\right)$. The first claim now follows from [FK18, proof of Lemma 1.3].

Since $C \subseteq \mathbf{P}^{g-1}$ is projectively normal, we have surjections $H^{0}\left(\widetilde{Z}, \mathcal{H}^{\otimes q}\right) \simeq H^{0}\left(Z, \mathcal{H}^{\otimes q}\right) \rightarrow$ $H^{0}\left(C, \omega_{C}^{\otimes q}\right)$ for all $q \geqslant 0$. The second claim then follows from the proof of the Green-Lefschetz theorem [Gre84a, Theorem 3.b.7].

For the final claim, we have $K_{p, 0}\left(\widetilde{Z},-\iota^{*}(x+y) ; \mathcal{H}\right)=0$. Taking Koszul cohomology of the short exact sequence (4.1) yields the exact sequence

$$
0 \rightarrow K_{p, 1}(\widetilde{Z}, \mathcal{H}) \rightarrow \mathcal{K}_{p, 1}(\mathbb{M}, \mathcal{S}) \stackrel{\delta}{\rightarrow} K_{p-1,1}\left(\widetilde{Z},-\iota^{*}(x+y) ; \mathcal{H}\right) \rightarrow K_{p-1,2}(\widetilde{Z}, \mathcal{H})
$$

The claim then follows from the previous statements.

We have a short exact sequence

$$
0 \rightarrow \mathcal{O}_{D} \rightarrow \nu_{*} \mathcal{O}_{C} \rightarrow \mathcal{O}_{p} \rightarrow 0
$$

inducing injective maps

$$
H^{0}\left(D, \omega_{D}^{\otimes q}\right) \hookrightarrow H^{0}\left(C, \omega_{C}^{\otimes q}(q x+q y)\right), \quad q \geqslant 0,
$$

which are isomorphisms for $q=0,1$. Since we may write

$$
K_{p, 1}\left(D, \omega_{D}\right) \simeq \operatorname{Ker}\left(\wedge^{p} H^{0}\left(\omega_{D}\right) \otimes H^{0}\left(\omega_{D}\right) / \wedge^{p+1} H^{0}\left(\omega_{D}\right) \rightarrow \wedge^{p-1} H^{0}\left(\omega_{D}\right) \otimes H^{0}\left(\omega_{D}^{\otimes 2}\right)\right),
$$

and likewise for $K_{p, 1}\left(C, \omega_{C}(x+y)\right)$, we have a canonical isomorphism

$$
K_{p, 1}\left(C, \omega_{C}(x+y)\right) \simeq K_{p, 1}\left(D, \omega_{D}\right)
$$

for any $p$.

Our goal is to show that the map $\delta: K_{p, 1}\left(C, \omega_{C}(x+y)\right) \rightarrow K_{p-1,1}\left(\widetilde{Z},-\iota^{*}(x+y) ; \mathcal{H}\right)$ is injective for $p=g-k+1$ under certain hypotheses, which then implies $K_{g-k+1,1}\left(C, \omega_{C}\right)=0$. To proceed, we need to use the Eagon-Nothcott resolution of a scroll [Sch86, §1]. Let

$$
\mathcal{E}=\mathcal{O}_{\mathbf{P}^{1}}\left(e_{1}\right) \oplus \cdots \oplus \mathcal{O}_{\mathbf{P}^{1}}\left(e_{d}\right), \quad e_{i}>0 \text { for all } i
$$

be a globally generated vector bundle of rank $d$ on $\mathbf{P}^{1}$, and let

$$
X:=j(\mathbf{P}(\mathcal{E})) \subseteq \mathbf{P}^{r},
$$




\section{ProjeCting SYZYGIES OF CURVES}

where $r=h^{0}\left(\mathcal{O}_{\mathbf{P}(\mathcal{E})}(1)\right)-1$ is the associated ruled variety of dimension $d$ and minimal degree $r-d+1$, and where $j: \mathbf{P}(\mathcal{E}) \hookrightarrow \mathbf{P}\left(H^{0}\left(\mathcal{O}_{\mathbf{P}(\mathcal{E})}(1)\right)\right)$ is the natural morphism.

Let $p \in X$ be a smooth point, and consider the projection

$$
\pi_{p}: \mathbf{P}^{r} \rightarrow \mathbf{P}^{r-1} \text {. }
$$

Let $Y \subseteq \mathbf{P}^{r}$ denote the cone over the image $\pi_{p}(X)$, with vertex at $p$. Then $X \subseteq Y$, and the cone $Y$ is a variety of minimal degree of dimension 1 higher than $X$. We may resolve $Y$ by a smooth rational normal scroll $\tilde{Y}$; see [EH87]. We have a birational morphism $B l_{p}(Y) \rightarrow \tilde{Y}$ from the blow-up of $Y$ at $p$ to $\widetilde{Y}$. Let $\mu: \widetilde{Y} \rightarrow Y$ denote the resolution of singularities, and let $X^{\prime} \subseteq \widetilde{Y}$ be the strict transform of $X$. Let $\mathcal{H}$ and $\mathcal{R}$ denote the classes of the hyperplane and ruling of $\widetilde{Y}$, respectively. These classes span $\operatorname{Pic}(\widetilde{Y})$.

Lemma 4.4. We have $\mathcal{O}_{\widetilde{Y}}\left(X^{\prime}\right) \simeq \mathcal{O}_{\widetilde{Y}}(\mathcal{H}+\mathcal{R})$.

Proof. Let $a$ and $b$ be such that $\mathcal{O}_{\widetilde{Y}}\left(X^{\prime}\right) \simeq \mathcal{O}_{\widetilde{Y}}(a \mathcal{H}+b \mathcal{R})$. Firstly, the image of the ruling on the scroll $\mathbf{P}(\mathcal{E})$ gives the ruling on $\pi_{p}(X)$ under the map $\pi_{p} \circ j$, and this pulls back to the ruling $\mathcal{R}$ on $\tilde{Y}$ under $\pi_{p} \circ \mu$. We see from this that $X^{\prime}$ meets a general ruling $\mathcal{R}$ of $\tilde{Y}$ in a linear space of codimension 1, and so $a=1$. We have $\operatorname{deg}(\widetilde{Y})=\operatorname{deg}\left(\pi_{p}(X)\right)=\operatorname{deg}(X)-1$. Thus, if $d=\operatorname{deg}(\widetilde{Y})$, then $d+1=\mathcal{H}^{\operatorname{dim}(X)} \cdot(a \mathcal{H}+b \mathcal{R})=a d+b$, and so $b=(1-a) d+1$, which gives $a=1$ and $b=1$.

By Lemma 4.4, we have a short exact sequence

$$
0 \rightarrow \mathcal{O}_{\widetilde{Y}}(-\mathcal{H}-\mathcal{R}) \rightarrow \mathcal{O}_{\widetilde{Y}} \rightarrow \mathcal{O}_{X^{\prime}} \rightarrow 0
$$

Notice that, by the Leray spectral sequence applied to $\widetilde{Y} \rightarrow \mathbf{P}^{1}$, we have $H^{1}\left(\widetilde{Y}, \mathcal{H}^{\otimes q-1}(-\mathcal{R})\right)=0$ for $q \geqslant 0$. Thus we have a short exact sequence

$$
0 \rightarrow \bigoplus_{q \in \mathbb{Z}} H^{0}\left(\tilde{Y}, \mathcal{H}^{\otimes q-1}(-\mathcal{R})\right) \rightarrow \bigoplus_{q \in \mathbb{Z}} H^{0}\left(\tilde{Y}, \mathcal{H}^{\otimes q}\right) \rightarrow \bigoplus_{q \in \mathbb{Z}} H^{0}\left(X^{\prime}, \mathcal{H}^{\otimes q}\right) \rightarrow 0
$$

of $\operatorname{Sym}\left(H^{0}(\widetilde{Y}, \mathcal{H})\right)$-modules. This induces a long exact sequence

$$
\begin{aligned}
0 & =K_{p, 0}(\widetilde{Y},-\mathcal{R} ; \mathcal{H}) \rightarrow K_{p, 1}(\widetilde{Y}, \mathcal{H}) \rightarrow K_{p, 1}\left(X^{\prime}, \mathcal{H}\right) \\
& \stackrel{\Delta}{\rightarrow} K_{p-1,1}(\tilde{Y},-\mathcal{R} ; \mathcal{H}) \rightarrow K_{p-1,2}(\widetilde{Y}, \mathcal{H}) \rightarrow \cdots
\end{aligned}
$$

Lemma 4.5. Set $f=r-d+1=\operatorname{deg}(X)$. Then

$$
\Delta: K_{f-1,1}\left(X^{\prime}, \mathcal{H}\right) \stackrel{\sim}{\rightarrow} K_{f-2,1}(\widetilde{Y},-\mathcal{R} ; \mathcal{H})
$$

is an isomorphism for $p=f-1$.

Proof. The Eagon-Northcott complex provides minimal resolutions for the scrolls $X^{\prime}$ and $\tilde{Y}$; see [EN62] and [Sch86]. These resolutions have length 1 less than the degree of the variety, and all matrices between terms in the resolution have linear forms as entries, with the exception of the first map, which has quadratic entries. Thus $K_{f-1,1}(\widetilde{Y}, \mathcal{H})=K_{f-2,2}(\widetilde{Y}, \mathcal{H})=0$.

We now arrive at the main result of this section. 


\section{KEMENY}

Theorem 4.6. Let $D$ be the 1-nodal $k$-gonal curve as above, with normalization the smooth curve $C$ of genus $g$ and line bundle $B \in W_{k}^{1}(D)$ satisfying $\nu^{*} B \simeq A$, for $k \geqslant 3$. Assume

(i) $h^{0}\left(C, A^{\otimes 2}\right)=3$ and

(ii) $b_{g+1-k, 1}\left(D, \omega_{D}\right)=g+1-k$.

Then $K_{g+1-k, 1}\left(C, \omega_{C}\right)=0$.

Proof. By Proposition 4.3, it is equivalent to show that the boundary map

$$
\delta: K_{g+1-k, 1}\left(C, \omega_{C}(x+y)\right) \rightarrow K_{g-k, 1}\left(\widetilde{Z},-\iota^{*}(x+y) ; \mathcal{H}\right)
$$

is injective. Consider the scroll

$$
X=\bigcup_{s \in|B|}\langle s\rangle \subseteq \mathbf{P}^{g}
$$

induced by the given $g_{k}^{1}$ on $D$; see [Sch86, §2]. The condition $h^{0}\left(A^{\otimes 2}\right)=3$ implies $h^{0}\left(B^{\otimes 2}\right)=3$. Then $X$ has degree $f=g+2-k$ and, further, is smooth since $h^{0}\left(D, B^{\otimes 2}\right)=3$; see [FK19, $\left.\S 4\right]$. As above, let $Y$ denote the cone over $\pi_{p}(X)$ with vertex at $p$, where $p \in D$ is the node and

$$
\pi_{p}: \mathbf{P}^{g} \rightarrow \mathbf{P}^{g-1}
$$

is the projection away from $p$. Further, let $\mu: \tilde{Y} \rightarrow Y$ denote the resolution of singularities, and let $X^{\prime} \subseteq \widetilde{Y}$ denote the strict transform of $X$.

Let $Z \subseteq \mathbf{P}^{g}$ denote the cone over $C \simeq \pi_{p}(D)$ with vertex at $p$, and let $\widetilde{\nu}: \widetilde{Z} \rightarrow Z$ be the desingularization. We have a natural diagram

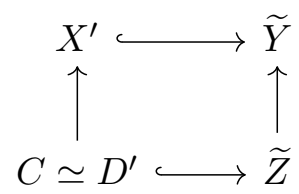

relating the strict transforms $D^{\prime}$ and $X^{\prime}$ and the desingularizations $\widetilde{Y}$ and $\widetilde{Z}$ of the cones over the projections. Pulling back the class of the ruling $\mathcal{R}$ on $\widetilde{Y}$ to $\widetilde{Z}$ yields $\iota^{*} A$, where

$$
\iota: \widetilde{Z} \simeq \mathbf{P}\left(\mathcal{O}_{C} \oplus \omega_{C}\right) \rightarrow C
$$

is the projection, as above. Let $T$ be the unique element of $|A|$ passing through $x$ and $y$. We have a commutative diagram of short exact sequences

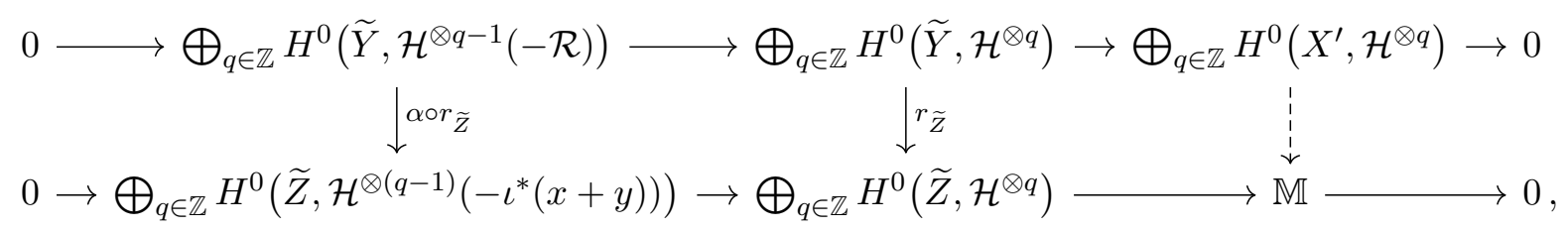

where the maps $r_{\widetilde{Z}}$ are induced by pull-back, where $\alpha$ is induced by multiplication by $\iota^{*} T(-x-y)$, where the lower row is the short exact sequence (4.1) defined earlier, and where the dashed arrow is the induced map. Note that, by Lemmas 4.2 and 4.4, the pull-back of the divisor $X^{\prime}$ to $\widetilde{Z}$ is $D^{\prime} \cup \iota^{-1}(T(-x-y))$. 


\section{ProjeCting SYZYGIES OF CURVES}

Upon taking Koszul cohomology, we get the commutative diagram

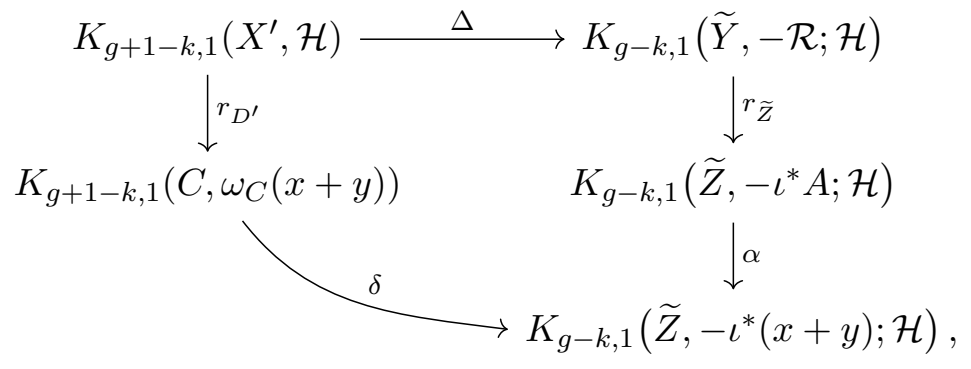

where $\Delta$ is an isomorphism by Proposition 4.5. It thus suffices to show that $r_{D^{\prime}}$ is an isomorphism, whereas $\alpha$ and $r_{\widetilde{Z}}$ are injective.

To see that $r_{D^{\prime}}$ is an isomorphism, first note that since we have birational morphisms $B l_{p} X \rightarrow$ $X^{\prime} \rightarrow X$, we have isomorphisms $H^{0}\left(B l_{p} X, q \mathcal{H}\right) \simeq H^{0}\left(X^{\prime}, q \mathcal{H}\right) \simeq H^{0}(X, q \mathcal{H})$ for all $q$ and so

$$
K_{p, 1}\left(X^{\prime}, \mathcal{H}\right) \simeq K_{p, 1}(X, \mathcal{H}) .
$$

Next, $r_{D^{\prime}}$ is injective by the same proof as that of [FK19, Lemma 4.4] (note $D^{\prime} \subseteq B l_{p} X$ ). From the Eagon-Northcott resolution, we see $b_{g+1-k, 1}(X, \mathcal{H})=f-1=g+1-k$. Hence the claim follows from the assumption $b_{g+1-k, 1}\left(D, \omega_{D}\right)=g+1-k$ since $K_{p, 1}\left(D, \omega_{D}\right) \simeq K_{p, 1}\left(C, \omega_{C}(x+y)\right)$ for all $p$.

The injectivity of $\alpha$ follows from the commutative diagram

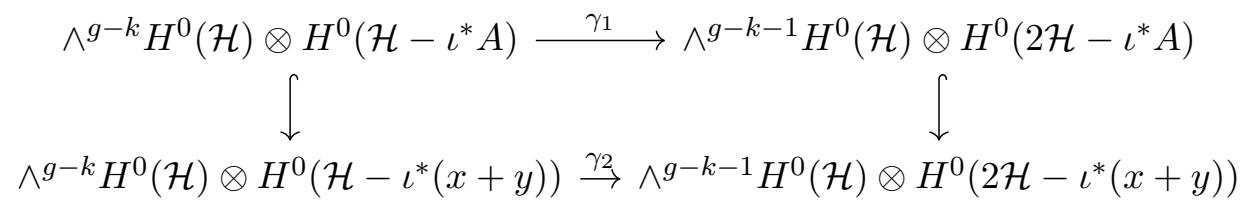

since $\alpha$ is the induced map $\operatorname{Ker}\left(\gamma_{1}\right) \hookrightarrow \operatorname{Ker}\left(\gamma_{2}\right)$.

It remains to show that $r_{\widetilde{Z}}$ is injective. To begin, the commutative diagram

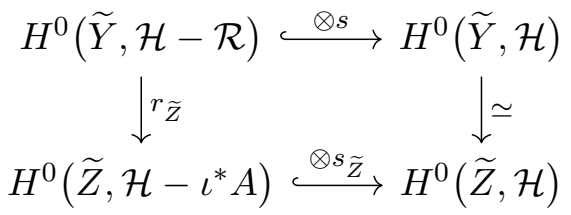

for general $s \in|\mathcal{R}|$ shows that the pull-back map $r_{\widetilde{Z}}: H^{0}(\widetilde{Y}, \mathcal{H}-\mathcal{R}) \hookrightarrow H^{0}\left(\widetilde{Z}, \mathcal{H}-\iota^{*} A\right)$ is injective. Next, the Eagon-Northcott resolution of

$$
\Gamma_{\widetilde{Y}}(\mathcal{H} ;-\mathcal{R}):=\bigoplus_{q \in \mathbb{Z}} H^{0}(\widetilde{Y}, q \mathcal{H}-\mathcal{R})
$$

as described in [Sch86, $\S 1.5$, p. 111], is 1-linear; that is, $K_{p, q}(\widetilde{Y},-\mathcal{R} ; \mathcal{H})=0$ unless $q=1$ for all $p \geqslant 0$. In particular, $K_{p, 2}(\widetilde{Y},-\mathcal{R} ; \mathcal{H})=0$ for all $p \geqslant 0$. Letting $M_{\mathcal{H}}$ be the kernel bundle

$$
0 \rightarrow M_{\mathcal{H}} \rightarrow H^{0}(\mathcal{H}) \otimes \mathcal{O}_{\widetilde{Y}} \rightarrow \mathcal{H}_{\widetilde{Y}} \rightarrow 0
$$

this implies $H^{1}\left(\widetilde{Y}, \wedge^{p} M_{\mathcal{H}}(\mathcal{H}-\mathcal{R})\right)=0$ for all $p \geqslant 0$, by the kernel bundle description of Koszul cohomology [AN10] (note that we have already observed $H^{1}(\widetilde{Y}, \mathcal{H}-\mathcal{R})=0$ ). For all $p \geqslant 0$, we 


\section{KEMENY}

have the short exact sequence

$$
0 \rightarrow \bigwedge^{p} M_{\mathcal{H}} \otimes(\mathcal{H}-\mathcal{R}) \rightarrow \bigwedge^{p} H^{0}(\widetilde{Y}, \mathcal{H}) \otimes(\mathcal{H}-\mathcal{R}) \rightarrow \bigwedge^{p-1} M_{\mathcal{H}} \otimes(2 \mathcal{H}-\mathcal{R}) \rightarrow 0
$$

and canonical isomorphisms

$$
\begin{aligned}
K_{p, 1}(\tilde{Y},-\mathcal{R} ; \mathcal{H}) & \simeq H^{0}\left(\tilde{Y}, \bigwedge^{p} M_{\mathcal{H}} \otimes(\mathcal{H}-\mathcal{R})\right) \\
K_{p, 1}\left(\widetilde{Z},-\iota^{*} A ; \mathcal{H}\right) & \simeq H^{0}\left(\widetilde{Z}, \bigwedge^{p} M_{\mathcal{H}} \otimes\left(\mathcal{H}-\iota^{*} A\right)\right) .
\end{aligned}
$$

There is a commutative diagram

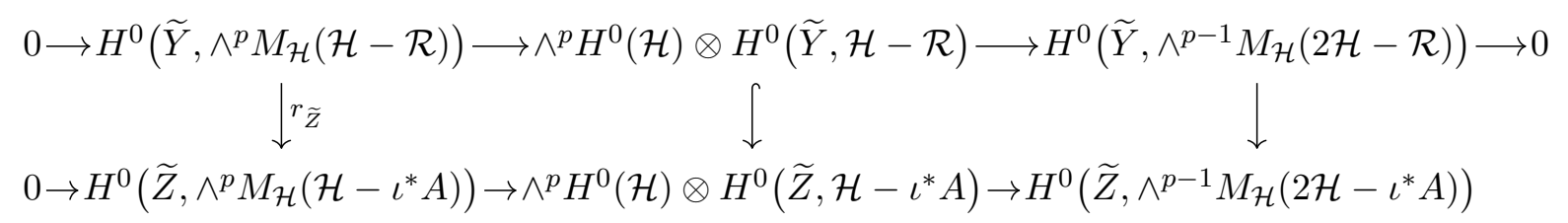

with exact rows. The claim now follows from the snake lemma.

We end this section by proving a result on Green's conjecture for curves of even genus and maximal gonality.

TheOrem 4.7. Let $C$ be a smooth curve of genus $g=2 n$ and gonality $k=n+1$. Suppose that for $x, y \in C$ general, there is at most one $A \in W_{n+1}^{1}(C)$ such that $A(-x-y)$ is effective. Further assume $h^{0}\left(C, A^{\otimes 2}\right)=3$. Then $C$ satisfies Green's conjecture.

Proof. If, for $x, y \in C$ general, there is no $A \in W_{n+1}^{1}(C)$ with $A(-x-y)$ effective, then $\operatorname{dim} W_{n+1}^{1}(C)=0$ and the result follows from [Apr05]. So we may assume that there is precisely one $A \in W_{n+1}^{1}(C)$ such that $A(-x-y)$ is effective. Let $D$ be the 1-nodal curve of genus $2 n+1$ obtained by identifying $x$ and $y$, and let $\nu: C \rightarrow D$ be the normalization. There is a line bundle $B$ on $D$ of degree $n+1$ and with $h^{0}(D, B)=2$ such that $\nu^{*} B \simeq A$. Further, $h^{0}\left(C, A^{\otimes 2}\right)=3$ implies that $h^{0}\left(D, B^{\otimes 2}\right)=3$ (we have $h^{0}\left(D, B^{\otimes 2}\right) \geqslant 3$ by the base-point-free pencil trick). Note further that if $T \in \overline{\operatorname{Jac}}(D)$ is a rank 1 torsion-free sheaf of degree $n+1$ with $h^{0}(D, T) \geqslant 2$, then we must have $T \simeq B$. Indeed, such a $T$ must be locally free, or else $T \simeq \nu_{*} T^{\prime}$ for $T^{\prime} \in W_{n}^{1}(C)$, contradicting that $C$ has gonality $n$, and then $T$ must be $B$ from the assumption that there is at most one $A \in W_{n+1}^{1}(C)$ such that $A(-x-y)$ is effective. By [FK19, Remark 3.2], we have $b_{n, 1}\left(D, \omega_{D}\right)=n$. From Theorem 4.6, we deduce that $C$ satisfies Green's conjecture.

\section{Green's conjecture for elliptic covers}

In this section, we prove Green's conjecture for general elliptic covers. We first fix some notation. Let $E$ denote a smooth elliptic curve, with polarization $L=\mathcal{O}_{E}(p)$ for $p \in E$ a fixed point. We denote by $\mathcal{M}_{g}\left(E, d_{1}\right)$ the moduli space of stable maps $f: C \rightarrow E$ with $C$ smooth of genus $g \geqslant 2$ and $\operatorname{deg}\left(f^{*} L\right)=d_{1} \geqslant 1$, and likewise let $\mathcal{M}_{g}\left(\mathbf{P}^{1}, d_{2}\right)$ denote the moduli space of degree $d_{2} \geqslant 1$ genus $g$ stable maps to $\mathbf{P}^{1}$. We lastly write

$$
\mathcal{M}_{g}\left(E \times \mathbf{P}^{1}, d_{1}, d_{2}\right)
$$

for the Deligne-Mumford stack of stable maps $f: C \rightarrow E \times \mathbf{P}^{1}$ with $\operatorname{deg}\left(f^{*} L\right)=d_{1}$ and $\operatorname{deg} f^{*} \mathcal{O}_{\mathbf{P}^{1}}(1)=d_{2}$. 


\section{ProjeCting Syzygies OF CURVES}

LEMma 5.1. Fix $g \geqslant 2$. The stacks $\mathcal{M}_{g}\left(E, d_{1}\right)$ and $\left.\mathcal{M}_{g}\left(\boldsymbol{P}^{1}, d_{2}\right)\right)$ are smooth of respective dimensions $2 g-2$ and $2 g-2+2 d_{2}$.

Proof. For any smooth projective variety $X$ and non-constant stable map $f: C \rightarrow X$ from a smooth curve $C$, the deformation theory of $f$ is determined by the normal sheaf $N_{f}$, see $[\mathrm{BHT} 11, \S 4]$, which fits into the short exact sequence

$$
0 \rightarrow T_{C} \stackrel{d f}{\rightarrow} f^{*} T_{X} \rightarrow N_{f} \rightarrow 0 .
$$

In particular, when $\operatorname{dim} X=1$, the sheaf $N_{f}$ is supported on the ramification locus of $f$, and so $h^{1}\left(C, N_{f}\right)=0$. Thus $\mathcal{M}_{g}\left(E, d_{1}\right)$ and $\mathcal{M}_{g}\left(\mathbf{P}^{1}, d_{2}\right)$ are smooth, with dimension at a point $f: C \rightarrow X$ given by $h^{0}\left(C, N_{f}\right)$, where $X \in\left\{E, \mathbf{P}^{1}\right\}$. For $X=E$, we have

$$
h^{0}\left(C, N_{f}\right)=\chi\left(\mathcal{O}_{C}\right)-\chi\left(\omega_{C}^{*}\right)=2 g-2,
$$

whereas for $X=\mathbf{P}^{1}$,

$$
h^{0}\left(C, N_{f}\right)=\chi\left(f^{*} \mathcal{O}_{\mathbf{P}^{1}}(2)\right)-\chi\left(\omega_{C}^{*}\right)=2 g-2+2 d_{2} .
$$

Proposition 5.2. With notation as above, let $\left[f: C \rightarrow E \times \boldsymbol{P}^{1}\right] \in \mathcal{M}_{g}\left(E \times \boldsymbol{P}^{1}, d_{1}, d_{2}\right)$, for $d_{1}, d_{2} \geqslant 1$, be a point such that $f$ is birational to its image. Then each component of $\mathcal{M}_{g}(E \times$ $\left.\boldsymbol{P}^{1}, d_{1}, d_{2}\right)$ containing $[f]$ is generically reduced and has dimension $g-1+2 d_{2}$.

Proof. We follow [AC81]. First of all, observe that each component $I$ of $\mathcal{M}_{g}\left(E \times \mathbf{P}^{1}, d_{1}, d_{2}\right)$ containing $[f]$ has dimension at least

$$
\operatorname{dim} \mathcal{M}_{g}\left(E, d_{1}\right)+\mathcal{M}_{g}\left(\mathbf{P}^{1}, d_{2}\right)-\operatorname{dim} \mathcal{M}_{g}=g-1+2 d_{2} .
$$

Let $\left[h: C^{\prime} \rightarrow E \times \mathbf{P}^{1}\right] \in I$ be a general point. The normal sheaf $N_{h}$ of the morphism $h$ fits into an exact sequence

$$
0 \rightarrow K_{h} \rightarrow N_{h} \rightarrow N_{h}^{\prime} \rightarrow 0
$$

of sheaves on $C^{\prime}$, where $K_{h}$ is (non-canonically) isomorphic to $\mathcal{O}_{Z}$, where $Z$ is the ramification locus of $h$, and where $N_{h}^{\prime}$ is a line bundle. By [AC81, Lemma 1.4],

$$
h^{0}\left(N_{h}^{\prime}\right) \geqslant g-1+2 d_{2} \geqslant g+1 .
$$

For any line bundle $L$ on $C^{\prime}$, if $h^{1}(L) \neq 0$, then $|L|$ is a sublinear system of $\left|\omega_{C^{\prime}}\right|$ and hence $h^{0}(L) \leqslant g$. Thus $h^{1}\left(N_{h}^{\prime}\right)=0$ and hence $h^{1}\left(N_{h}\right)=0$, so that $I$ is smooth at $[h]$. Applying [AC81, Lemma 1.4] again, we may now conclude that $K_{h}=0$, that $h$ is unramified, and that $N_{h}$ is locally free of degree

$$
\operatorname{deg}\left(h^{*} T_{E \times \mathbf{P}^{1}}\right)+2 g-2=2 g-2+2 d_{2} .
$$

Thus $\operatorname{dim} I=h^{0}\left(N_{h}\right)=\chi\left(N_{h}\right)=g-1+2 d_{2}$, as required.

We denote by $\mathcal{H}_{g}^{E}\left(d_{1}\right) \subseteq \mathcal{M}_{g}\left(E, d_{1}\right)$ the open locus of primitive covers with simple ramification. The space $\mathcal{H}_{g}^{E}\left(d_{1}\right)$ is then non-empty and irreducible for $g \geqslant 2$, by a result of GabaiKazez [GK87] (see also [Buj14]).

For a smooth curve $C$, let $W_{k}^{1}(C)$ be the Brill-Noether variety of line bundles $L$ of degree $k$ with at least two sections, and let $G_{d}^{1}(C)$ be the variety whose closed points correspond to a $g_{d}^{1}$, that is, a pair of a line bundle $A \in W_{k}^{1}(C)$ together with a base-point-free linear system $V \subseteq H^{0}(A)$ of dimension 2, up to the natural PGL(2)-action [ACGH85]. If $C$ has gonality $k$, then elements of $W_{k}^{1}(C)=G_{k}^{1}(C)$ are called minimal pencils. 


\section{KEMENY}

Corollary 5.3. Let $[f: C \rightarrow E] \in \mathcal{H}_{g}^{E}\left(d_{1}\right)$ be a general point, let $(A, V) \in G_{d_{2}}^{1}(C)$, and suppose that $A$ is not isomorphic to $f^{*} B$ for some $B \in \operatorname{Pic}(E)$ with $\operatorname{deg}(B) \geqslant 2$. Then

$$
\operatorname{dim}_{[A]} G_{d_{2}}^{1}(C)=2 d_{2}-g-2 .
$$

In particular, if $2 d_{1} \leqslant\lfloor(g+3) / 2\rfloor$, then $C$ has gonality $2 d_{1}$, and if $2 d_{1}>\lfloor(g+3) / 2\rfloor$, then $C$ has gonality $\lfloor(g+3) / 2\rfloor$. Moreover, if $2 d_{1}<\lfloor(g+3) / 2\rfloor$, all minimal pencils of $C$ are of the form $f^{*} B$ with $B \in \operatorname{Pic}^{2}(E)$.

Proof. Let $(A, V) \in G_{d_{2}}^{1}(C)$ be base-point free, and suppose that $A$ is not the pull-back of a line bundle from $E$ of degree at least 2. Then $V$ induces a map $C \rightarrow \mathbf{P}^{1}$ of degree $d_{2}$, and we let $\left[h: C \rightarrow E \times \mathbf{P}^{1}\right] \in \mathcal{M}_{g}\left(E \times \mathbf{P}^{1}, d_{1}, d_{2}\right)$ be the product of this map with $f$. We claim that $h$ is birational to its image. Indeed, otherwise, let $D=h(C)$ be the image of $h$. Since $f$ is simply ramified and primitive, the projection to the first factor must induce an isomorphism $\mathrm{pr}_{1}: D \stackrel{\sim}{\rightarrow} E$. But then $A$ must be the pull-back of a line bundle $B$ from $E$ with $\operatorname{deg}(B) \geqslant 2$, which gives a contradiction. So $h$ is birational. Since we are assuming that $[f]$ is general, each component of $\mathcal{M}_{g}\left(E \times \mathbf{P}^{1}, d_{1}, d_{2}\right)$ containing [h] dominates $\mathcal{H}_{g}^{E}\left(d_{1}\right)$ under the natural forgetful morphism and all fibres have dimension

$$
g-1+2 d_{2}-(2 g-2),
$$

by Proposition 5.2. After subtracting $3=\operatorname{dim} \operatorname{PGL}(2)$, we see that each component of $G_{d_{2}}^{1}(C)$ containing $[A]$ has dimension $2 d_{2}-g-2$, as required. In particular, we must have $d_{2} \geqslant(g+2) / 2$. In fact, since $d_{2}$ is an integer, we have $d_{2} \geqslant\lfloor(g+3) / 2\rfloor$ (which is the gonality of a general curve of genus $g$ ). The remaining statements follow immediately.

We can now prove the main result of this section.

TheOREM 5.4. Let $[f: C \rightarrow E] \in \mathcal{H}_{g}^{E}\left(d_{1}\right)$ be a general point. Then Green's conjecture holds for $C$.

Proof. We may assume $d_{1} \geqslant 2$ as Green's conjecture holds for all elliptic curves. If $2 d_{1} \geqslant$ $(g+3) / 2$, then by Corollary 5.3, the curve $C$ has maximal gonality $k:=\lfloor(g+3) / 2\rfloor$ and, further, $\operatorname{dim} G_{k+n}^{1}(C) \leqslant n$ for $n \leqslant g+2-2 k$. Thus the statement follows from a theorem of HirschowitzRamanan [HR98] combined with Voisin's theorem [Voi02, Voi05] (in the odd genus case) and a theorem of Aprodu [Apr05, Theorem 2] (in even genus).

So it suffices to prove that for fixed $d_{1} \geqslant 2$ and all $g \geqslant 4 d_{1}-3$, the general point $[f: C \rightarrow E] \in$ $\mathcal{H}_{g}^{E}\left(d_{1}\right)$ satisfies $b_{g-2 d_{1}+1,1}\left(C, \omega_{C}\right)=0$, which further forces gon $(C)=2 d_{1}=\operatorname{Cliff}(C)+2$. We will prove this vanishing by induction, with the base case $g=4 d_{1}-3$ holding by the above. So suppose that $[f: C \rightarrow E] \in \mathcal{H}_{g}^{E}\left(d_{1}\right)$ is a general point, with $g \geqslant 4 d_{1}-3$, and suppose $b_{g-2 d_{1}+1,1}\left(C, \omega_{C}\right)=0$. Let $x, y \in C$ be distinct points such that $f(x)=f(y)$, and let $D$ be the curve of genus $g+1$ obtained by identifying $x$ and $y$. Let $g: D \rightarrow E$ be the unique morphism factoring through $f: C \rightarrow E$. By the Aprodu projection theorem (Theorem 4.1), we have $b_{g+2-2 d_{1}, 1}\left(D, \omega_{D}\right)=0$, which implies $b_{g+2-2 d_{1}, 1}\left(D^{\prime}, \omega_{D}^{\prime}\right)=0$ for a general point $\left[f^{\prime}: D^{\prime} \rightarrow E\right] \in \mathcal{H}_{g+1}^{E}\left(d_{1}\right)$ by the semicontinuity of Koszul cohomology.

\section{ACKNOWLEDGEMENTS}

We thank Juliette Bruce for an interesting discussion on projecting syzygies. We thank Gavril Farkas for suggesting that our results could be applied to Green's conjecture for curves of even genus and maximal gonality. 


\section{Projecting Syzygies OF CURVES}

\section{REFERENCES}

AC81 E. Arbarello and M. Cornalba, Footnotes to a paper of Beniamino Segre: "On the modules of polygonal curves and on a complement to the Riemann existence theorem" [Math. Ann. 100 (1928), 537-551], Math. Ann. 256 (1981), no. 3, 341-362; doi:10.1007/BF01679702.

ACGH85 E. Arbarello, M. Cornalba, P. A. Griffiths, and J. Harris, Geometry of algebraic curves, Vol. I, Grundlehren math. Wiss., vol. 267 (Springer-Verlag, New York, 1985); doi:10.1007/978-14757-5323-3.

AF11 M. Aprodu and G. Farkas, Green's conjecture for curves on arbitrary K3 surfaces, Compos. Math. 147 (2011), no. 3, 839-851; doi:10.1112/S0010437X10005099.

AF12 Green's conjecture for general covers, in Compact Moduli Spaces and Vector Bundles, Contemp. Math. vol. 564 (Amer. Math. Soc., Providence, RI, 2012), 211-226; doi:10.1090/ conm/564/11147.

$\mathrm{AFP}^{+} 19$ M. Aprodu, G. Farkas, Ş. Papadima, C. Raicu, and J. Weyman, Koszul modules and Green's conjecture, Invent. Math. 218 (2019), no. 3, 657-720; doi:10.1007/s00222-019-00894-1.

AN10 M. Aprodu and J. Nagel, Koszul cohomology and algebraic geometry, Univ. Lecture Ser., vol. 52 (Amer. Math. Soc., Providence, RI, 2010); doi:10.1090/ulect/052.

Apr02 M. Aprodu, On the vanishing of higher syzygies of curves, Math. Z. 241 (2002), no. 1, 1-15; doi:10.1007/s002090100403.

Apr05 Remarks on syzygies of d-gonal curves, Math. Res. Lett. 12 (2005), no. 2-3, 387-400; doi:10.4310/MRL.2005.v12.n3.a9.

BHT11 F. Bogomolov, B. Hassett, and Y. Tschinkel, Constructing rational curves on K3 surfaces, Duke Math. J. 157 (2011), no. 3, 535-550; doi:10.1215/00127094-1272930.

BS18 C. Bopp and F.-O. Schreyer, A version of Green's conjecture in positive characteristic, 2018, arXiv: 1803.10481.

Buj14 G. Bujokas, The Hurwitz space of covers of an elliptic curve $E$ and the severi variety of curves in $E \times \boldsymbol{P}^{1}, 2014$, arXiv:1409.0927.

CKK06 Y. Choi, P.-L. Kang, and S. Kwak, Higher linear syzygies of inner projections, J. Algebra 305 (2006), no. 2, 859-876; doi:10.1016/j.jalgebra.2006.08.007.

Cop88 M. Coppens, On G. Martens' characterization of smooth plane curves, Bull. London Math. Soc. 20 (1988), no. 3, 217-220; doi:10.1112/blms/20.3.217.

Cop97_ The existence of $k$-gonal curves possessing exactly two linear systems $g_{k}^{1}$, Math. Ann. 307 (1997), no. 2, 291-297; doi:10.1007/s002080050034.

EH87 D. Eisenbud and J. Harris, On varieties of minimal degree (a centennial account), Algebraic Geometry, Bowdoin, 1985 (Brunswick, Maine, 1985), Proc. Sympos. Pure Math., vol. 46 (Amer. Math. Soc., Providence, RI, 1987), 3-13; doi:10.1090/pspum/046.1/927946.

EL15 L. Ein and R. Lazarsfeld, The gonality conjecture on syzygies of algebraic curves of large degree, Publ. Math. Inst. Hautes Études Sci. 122 (2015), 301-313; doi:10.1007/s10240-015-0072-2.

EN62 J.A. Eagon and D. G. Northcott, Ideals defined by matrices and a certain complex associated with them, Proc. Roy. Soc. London Ser. A 269 (1962), 188-204; doi:10.1098/rspa.1962.0170.

EP99 D. Eisenbud and S. Popescu, Syzygy ideals for determinantal ideals and the syzygetic Castelnuovo lemma, Commutative Algebra, Algebraic Geometry, and Computational Methods (Hanoi, 1996) (Springer, Singapore, 1999), 247-258.

FHL84 W. Fulton, J. Harris, and R. Lazarsfeld, Excess linear series on an algebraic curve, Proc. Amer. Math. Soc. 92 (1984), no. 3, 320-322; doi:10.2307/2044824.

FK16 G. Farkas and M. Kemeny, The generic Green-Lazarsfeld secant conjecture, Invent. Math. 203 (2016), no. 1, 265-301; doi:10.1007/s00222-015-0595-7.

FK18 - The resolution of paracanonical curves of odd genus, Geom. Topol. 22 (2018), no. 7, 4235-4257; doi : 10.2140/gt.2018.22.4235. 


\section{KEMENY}

FK19 , Linear syzygies of curves with prescribed gonality, Adv. Math. 356 (2019), 106810; doi:10.1016/j.aim.2019.106810.

GK87 D. Gabai and W.H. Kazez, The classification of maps of surfaces, Invent. Math. 90 (1987), no. 2, 219-242; doi:10.1007/BF01388704.

Gre82 M. L. Green, The canonical ring of a variety of general type, Duke Math. J. 49 (1982), no. 4, 1087-1113; doi:10.1215/S0012-7094-82-04948-1.

Gre84a_, Koszul cohomology and the geometry of projective varieties, J. Differential Geom. 19 (1984), no. 1, 125-171; doi:10.4310/jdg/1214438426.

Gre84b_ Koszul cohomology and the geometry of projective varieties. II, J. Differential Geom. 20 (1984), no. 1, 279-289; doi:10.4310/jdg/1214439000.

Har77 R. Hartshorne, Algebraic geometry, Grad. Texts in Math., vol. 52 (Springer-Verlag, New YorkHeidelberg, 1977); doi:10.1007/978-1-4757-3849-0.

HR98 A. Hirschowitz and S. Ramanan, New evidence for Green's conjecture on syzygies of canonical curves, Ann. Sci. École Norm. Sup. (4) 31 (1998), no. 2, 145-152; doi:10.1016/S00129593(98)80013-X.

Kem18 M. Kemeny, Betti numbers of curves and multiple-point loci, 2018, arXiv:1804.09221.

Las78 A. Lascoux, Syzygies des variétés déterminantales, Adv. Math. 30 (1978), no. 3, 202-237; doi:10.1016/0001-8708(78) 90037-3.

Sch86 F.-O. Schreyer, Syzygies of canonical curves and special linear series, Math. Ann. 275 (1986), no. 1, 105-137; doi:10.1007/BF01458587.

Sch03 S Some topics in computational algebraic geometry, Advances in Algebra and Geometry (Hyderabad, 2001) (Hindustan Book Agency, New Delhi, 2003), 263-278.

Seg28 B. Segre, Sui moduli delle curve poligonali, e sopra un complemento al teorema di esistenza di Reimann, Math. Ann. 100 (1928), no. 1, 537-551; doi:10.1007/BF01448862.

SSW13 J. Schicho, F.-O. Schreyer, and M. Weimann, Computational aspects of gonal maps and radical parametrization of curves, Appl. Algebra Engrg. Comm. Comput. 24 (2013), no. 5, 313-341; doi:10.1007/s00200-013-0205-0.

Voi02 C. Voisin, Green's generic syzygy conjecture for curves of even genus lying on a K3 surface, J. Eur. Math. Soc. (JEMS) 4 (2002), no. 4, 363-404; doi:10.1007/s100970200042.

Voi05_, Green's canonical syzygy conjecture for generic curves of odd genus, Compos. Math. 141 (2005), no. 5, 1163-1190; doi:10.1112/S0010437X05001387.

Wey03 J. Weyman, Cohomology of vector bundles and syzygies, Cambridge Tracts in Math., vol. 149 (Cambridge Univ. Press, Cambridge, 2003); doi:10.1017/CB09780511546556.

Michael Kemeny michael.kemeny@gmail.com

University of Wisconsin-Madison, Department of Mathematics, 480 Lincoln Dr, WI 53706, USA 